\title{
A comparative, randomized study of levonorgestrel intrauterine system (LNG-IUS) vs Copper T 380 A intrauterine device applied during cesarean section
}

\author{
José A. López-Farfan ${ }^{1}$, Alicia Hernandez-Gonzalez ${ }^{1}$, Irvin J. Vélez-Machorro ${ }^{1}$, \\ Leopoldo A. Vázquez-Estrada ${ }^{2}$ \\ ${ }^{1}$ Department of Obstetrics and Gynecology, Regional Hospital of Puebla, Mexican Institute of Social Security, Puebla City, Mexico \\ ${ }^{2}$ Hospital Angeles Lindavista, Mexico City, Mexico \\ Email: leopoldo.vazquez@,bayer.com
}

Received 25 April 2012; revised 24 May 2012; accepted 1 June 2012

\begin{abstract}
Objective: To assess levonorgestrel-releasing intrauterine system LNG-IUS (Mirena ${ }^{\circledR}$ ) application at caesarean section (CS). Design: Randomized, comparative study. Setting: Department of obstetrics and gynecology in a primary reference hospital at Puebla City, Mexico. Sample: 396 women requiring CS signed informed consent and were randomly allocated to the post-placental application of LNG-IUS (198) or Copper T 380 A (198). Methods: Follow up visits at 6 weeks, and 6 and 12 months were performed. Main Outcomes Measures: IUDs expulsion, maternal and babies' health conditions, breastfeeding and menstrual patterns, adverse effects and pregnancies. Differences between groups were analyzed by Fisher and $\mathrm{X} 2$ tests, Odds ratios, relative risk and $95 \%$ confidence limits, as appropriate. Results: After one year of follow up, no pregnancies were reported. The IUD expulsion rate was $4.5 \%$ in each group. LNG-IUS users had a higher incidence of amenorrhea (OR 2.5 95\% CI 2.2 - 3) and menstrual patterns significantly brief and lighter than Copper T 380 A $(p<0.001)$ with lower incidence of dysmenorrhea (OR $0.195 \%$ CI 0.04 - 0.2). No detrimental effects of LNG-IUS on breastfeeding was observed and interestingly babies weights of LNG-IUS users was slightly above the average for age compared with Copper $T 380$ A users. This was probably related with a major proportion of women with normal ferritin serum levels $(94 \%$ vs $68 \%$ ) leading to better mother's general condition. Conclusions: LNG-IUS inserted during CS provides high efficacy contraception with additional benefits, mainly reducing menstrual bleeding and doing so, faster recovery of ferrous homeostasis after CS.
\end{abstract}

Keywords: Levonorgestrel-Releasing Intrauterine System; Medicated IUD; Copper T IUD; Cesarean
Section; Postpartum Contraception; Ferritin Levels

\section{INTRODUCTION}

During the postpartum time period, women are often highly motivated to initiate contraceptive use. Intrauterine device (IUD) insertion during this time period is an ideal method for some women, as it does not interfere with breastfeeding, is associated with less discomfort and fewer side effects than interval insertions and allows women to obtain safe, long-acting, highly effective contraception immediately after their obstetrical attention. Postpartum IUD insertion, however, may increase the risk of adverse events affecting safety (e.g., perforation, pain, bleeding) as well as effectiveness (i.e., expulsion). Nevertheless, post-placental placement during cesarean section is associated with lower expulsion rates when compared to immediate post-placental vaginal insertion. This immediate post-cesarean section insertion procedure was also found to be safe [1].

Concerns about local effects of levonorgestrel IUDs on uterine involution and about the theoretical risks of exposing breastfeeding infants to hormones released by these IUDs during the first 6 weeks postpartum have inhibited research into the consequences of their insertion during the postpartum period.

The levonorgestrel-releasing intrauterine system (LNGIUS) Mirena ${ }^{\circledR}$ primarily acts locally. According to the device's prescribing information, "in a study of 14 women using a LNG-IUS prototype during lactation, mean infant serum levels of levonorgestrel were approximately $7 \%$ of maternal serum levels" [2]. The LNG-IUS releases approximately $20 \mu \mathrm{g} /$ day initially, which decreases progressively to half that over the next 5 years. Very little levonorgestrel is absorbed into the maternal circulation. Levels are below that of progestin-only pills, which are safe in breastfeeding women. Shamash and cols. confirmed 
that levonorgestrel IUDs, inserted 6 - 8 weeks postpartum in lactating women provides highly effective and acceptable contraception and does not negatively influence breast-feeding or the growth and development of breastfed infants [3].

The potential clinical advantages of LNG-IUS at time of caesarean section (CS) have been described including: improvement of the puerperal uterine involution decreasing lochia and dysfunctional bleeding; induction of persistence of amenorrhea or oligomenorrhea after cessation of breast-feeding during 5 years of use, providing longterm and reversible contraception with effectiveness similar to that of female Sterilization. A brief audit from South Africa on LNG-IUS at cesarean section revealed the device was extremely well tolerated. There were no contraceptive failures, no complications and no expulsions of the intrauterine device. Patient satisfaction was extremely high [4].

Considering these, we decided to asses if LNG-IUS (levonorgestrel IUD) can be inserted during caesarean section designing a randomized study, comparing LNGIUS with our routinely clinical practice of Copper $\mathrm{T}$ Intrauterine Device (IUD) CS insertion.

\section{MATERIAL AND METHODS}

\subsection{Setting}

This study was conducted within the department of obstetrics and gynecology at the Mexican Institute of Social Security Regional Hospital of Puebla City, Mexico. The institutional review board approved the study.

\subsection{Sample}

Three hundred and ninety six women requiring CS were included and randomly allocated to receive LNG-IUS (198) or Copper T IUD (198). As routinely practice at our institution, an informed consent for the use of postpartum contraception is obtained during prenatal care visits. At this time specific informed consent for study and blinded randomization was also done using a randomization table from http://www.randomization.com. According to assignation number, an envelope with the type of IUD enclosed was stapled to the patient's file and open upon the time of delivery. The patients were not aware to which IUD were assigned as well as the investigator responsible of follow up. Exclusion criteria included: premature rupture of membranes, evidence or suspicion of chorioamnioitis and pre-partum hemorrhage. Patients' demographic data are shown in Table 1.

\subsection{Methods}

At Caesarean section and after removal of the placenta and before or during closure of the uterine wound, the
Table 1. Demographic characteristics.

\begin{tabular}{ccc}
\hline & LNG IUS & T Cu 380 \\
\hline Age (years \pm s.d.) & $24.8 \pm 5$ & $24.9 \pm 5.2$ \\
Cesarean indication (\%) & & \\
Cephalopelvic disproportion & 27 & 32 \\
Oligohidramnios & 17 & 15 \\
Fetal compromise & 24 & 28 \\
Others & 32 & 35 \\
Workers (\%) & 45 & 47 \\
\hline
\end{tabular}

devices were inserted manually, high in the uterine fundus orienting the IUD strings to the cervical os. At hospital discharge all subjects were provided with menstrual diaries, adverse event report forms and a prospect with information about IUD use and care. Follow up visits at the end of puerperium (6th week) and 6 and 12 months after insertion were performed. Participants underwent physical and pelvic examinations at each scheduled follow-up visit as well as the routinely care of their babies. Subjects returned their daily bleeding and side effect diaries in each visit. The analysis of the bleeding pattern was done using 90-day reference periods.

For the analysis of the bleeding pattern, the following definitions were used: amenorrhea was defined as no bleeding or spotting throughout the reference period. Infrequent bleeding was defined as fewer than three bleeding-spotting episodes (B-S) starting within a reference period, excluding amenorrhea. Frequent bleeding was defined as more than five B-S episodes starting within a reference period and prolonged bleeding was defined by at least one B-S episode starting within a reference period and lasting $>14$ days. A B-S episode was defined as any set of $>1$ consecutive bleeding or spotting days bounded at each end by at least 1 bleeding-free day.

In a subset of patients $(n=100)$ blood venous samples were taken at the end of the observational period to evaluate serum ferritin levels. Normal ferritin value was defined as $>15 \mathrm{ng} / \mathrm{ml}$. Additional in-person visits included interim visits for clinical issues or desired early termination from the study. Participants were discontinued from the study if they became pregnant or if the device was expelled, displaced downward (such that the device appeared at the cervical os on speculum exam) or removed for any reason. Differences between groups were analyzed by Fisher and X2 tests, Odds ratios, relative risk and $95 \%$ confidence limits as appropriate.

\section{RESULTS}

At the end of the study no pregnancies were reported Pearl Index $=0\left({ }_{95 \%} \mathrm{CI} 0-0.2\right)$. Discontinuation was as 
follows: for LNG-IUS group $18.2 \%(\mathrm{n}=36)$ and for Copper T IUD group 20.7\% $(\mathrm{n}=41)$ after 12 months. The reasons for discontinuation are presented together with numbers and percentages in Table 2 . The majority of women, who discontinued the method, besides loss of follow-up, did so because of spontaneous IUD expulsion (4.5\% in each group) and unacceptable bleeding pattern: amenorrhea and spotting for $2 \%$ of LNG-IUS users and excessive bleeding in $3.5 \%$ of Copper T IUD users.

\subsection{Bleeding Patterns}

From the total study population, 320 women contributed at least one reference period for analysis. Bleeding patterns are shown in Figure 1.

In LNG-IUS group, $65 \%$ of users had amenorrhea at 6 months, and $48 \%$ at the 12 th month of following-up. The second bleeding pattern in LNG-IUS group was infre-

Table 2. Reasons for discontinuation.

\begin{tabular}{ccccc}
\hline \multirow{2}{*}{ Reason } & \multicolumn{2}{c}{ LNG IUS } & \multicolumn{2}{c}{ Copper T IUD } \\
\cline { 2 - 5 } & $\mathbf{n}$ & $\mathbf{\%}$ & $\mathbf{n}$ & $\mathbf{\%}$ \\
\hline Hypermenorrhea & 0 & 0.0 & 7 & 3.5 \\
Dysmenorrhea & 0 & 0.0 & 1 & 0.5 \\
Spotting & 2 & 1.0 & 0 & 0.0 \\
Pelvic pain & 2 & 1.0 & 0 & 0.0 \\
PID* & 1 & 0.5 & 1 & 0.5 \\
Cervicitis & 0 & 0.0 & 1 & 0.5 \\
Amenorrhea & 2 & 1.0 & 0 & 0.0 \\
Weight gain & 1 & 0.5 & 0 & 0.0 \\
Expulsion & 9 & 4.5 & 9 & 4.5 \\
Loss to follow-up & 19 & 9.6 & 22 & 11.1 \\
Total & $\mathbf{3 6}$ & $\mathbf{1 8 . 2}$ & $\mathbf{4 1}$ & $\mathbf{2 0 . 7}$ \\
\hline
\end{tabular}

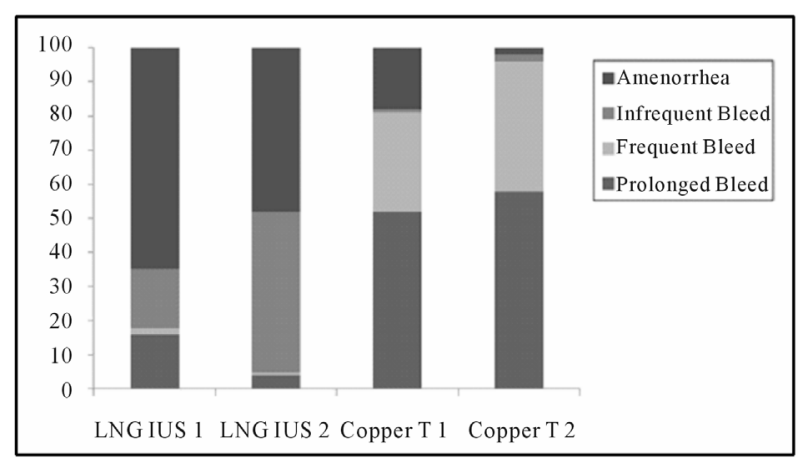

Figure 1. Bleeding patterns along the study. In order to facilitate their interpretation, the four 90 periods of reference of study observation are analyzed together as 2 periods of 180 days (period $1=$ first 6 months and period $2=$ last 6 months). quent bleeding present in $17 \%$ of users in the first semester and in $47 \%$ in the last reference period.

For Copper T IUD group, prolonged bleeding was predominant as a result of $52 \%$ of patients reporting this pattern in the first semester enhancing to $59 \%$ at the 12 th month. Frequent bleeding was present in $30 \%$ and $37 \%$ of patients in respectively reference periods.

\subsection{Ferritin Levels}

At the end of study, normal Ferritin serum levels $(>15$ $\mathrm{ng} / \mathrm{ml})$ were detected in $94 \%$ of LNG-IUS ${ }^{\circledR}$ users $(\mathrm{n}=5$ $0)$ and in $68 \%$ of Copper T IUD users $(n=50) p<0.001$.

\subsection{Breastfeeding}

Breastfeeding were supplied by $93 \%$ of LNG-IUS $^{\circledR}$ and $94 \%$ of Copper T IUD users during a mean of 6.98 months, $67.5 \%$ of total sample from 3 to 12 months. There were no significant differences between groups.

Figure 2, shows Breastfed infants weights Nomogram along the first year of life. The median weight at birth for LNG-IUS users was $3.0 \mathrm{Kg}$ (minimum 1.3, maximum 4.8) and for Copper T IUD was $3.1 \mathrm{Kg}$ (min. 1.0, max. 4.4) $\mathrm{p}$ $=0.7$. At sixth month the median weight in LNG-IUS group was $8.0 \mathrm{Kg}$ (min. 6.2, max. 9.0) and for Copper $\mathrm{T}$ IUD was $7.5 \mathrm{Kg}$ (min. 6.0, max. 9.0) respectively $\mathrm{p}<$ 0.0001 . At twelfth month the median weight for LNGIUS group reached $10 \mathrm{Kg}$ (min. 7.1, max. 12.5) and for Copper T IUD was $9.7 \mathrm{Kg}$ (min. 6.9, max. 12.5) $\mathrm{p}<$ 0.0001 .

Table 3 shows the number and rate of adverse events. Significant differences between groups were noticed, mainly higher incidence of women's weight increase (OR 1.4 95\% CI 1.2 - 1.8) and lower incidence of dysmenorrhea (OR $0.195 \%$ CI $0.04-0.2$ ) and vaginitis (OR $0.495 \%$ CI $0.3-0.7)$ in the LNG-IUS group. Two cases of Pelvic Inflammatory disease occurred in each one of the study groups, far from puerperium, and were cata-

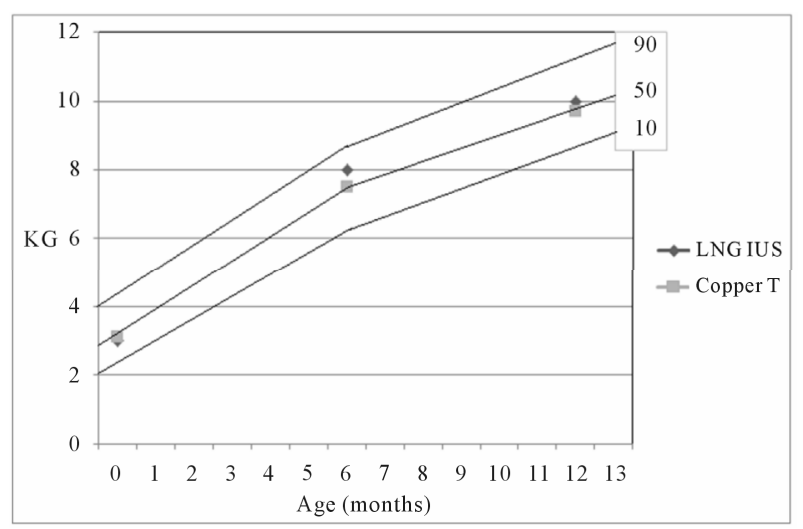

Figure 2. Breastfed infants weights Nomogram. The lines represent de $90^{\text {th }}, 50^{\text {th }}$ and $10^{\text {th }}$ percentiles. 
Table 3. Adverse events (AE) along the study.

\begin{tabular}{cccccc}
\hline \multirow{2}{*}{ AE } & \multicolumn{4}{c}{ LNG IUS } & \multicolumn{4}{c}{ Copper T IUD } \\
\cline { 2 - 6 } & $\mathbf{n}$ & $\mathbf{\%}$ & $\mathbf{n}$ & $\mathbf{\%}$ & $\mathbf{p}$ \\
\hline Weight increase & 158 & 79.8 & 97 & 49.0 & $<0.05$ \\
Dysmenorrhea & 6 & 3.0 & 47 & 23.7 & $<0.01$ \\
Mastalgia & 13 & 6.6 & 4 & 2.0 & 0.2 \\
Vaginits & 45 & 22.7 & 81 & 40.9 & $<0.05$ \\
Cervicitis & 3 & 1.5 & 5 & 2.5 & 0.3 \\
Pelvic infection & 1 & 0.5 & 1.0 & 0.5 & n.s. \\
\hline
\end{tabular}

logued as serious adverse event and related with IUD use. Both cases were discontinued from study, and properly treated with antibiotic therapy before IUD extraction.

\section{DISCUSSION}

The postpartum period is a convenient time during a woman's life to have an IUD inserted, since it may be one of the few times she is in contact with medical services. Mexico has committed major resources and programmatic attention to postpartum IUD programs. In the Mexican Social Security System, (Instituto Mexicano del Seguro Social), delivery-room staff are trained to insert IUDs, and women are counseled during prenatal care about postpartum contraceptive options. IUDs are the most popular method of postpartum contraception in Mexico [5]. Studies in China, Belgium, and Mexico, examining IUD insertion through the abdominal incision immediately after cesarean delivery, have found the procedure to be safe and expulsion rates to be low [6-10]. At our institution the insertion of Copper T 380 A IUD is a routinely practice at the time of Cesarean Section; furthermore is a good contraceptive method for a lactating woman because it has no effect on the quantity or composition of breast milk [11,12]. With the LNG-IUS, small amounts of progestin are found in breast milk, although these low levels apparently do not affect child health $[13,14]$.

In the present study, no negative effects were detected on breast-feeding performance with the use of LNG-IUS during the first year of lactation. Interestingly there was a slight difference of growth pattern between babies favoring LNG-IUS group. We consider that at least this is not a concern about babies' health and on the contrary these data may support the hypothesis that a faster and better recovery of ferrous homeostasis after CS in LNGIUS group based in a major proportion of women with normal Ferritin levels, related with extremely low incidence of menstrual bleeding leading to mothers better general health conditions.

This menstrual pattern induced by LNG-IUS may represent an advantage specifically after CS because it has been reported [15] that the mean hemorrhage after vaginal delivery is $373 \mathrm{ml}(95 \%$ CI 370 - 376) but after cesarean section, the mean hemorrhage is significantly higher $(517 \mathrm{ml} ; 95 \% \mathrm{CI} 503$ - 530) and more women experienced severe hemorrhage (5.9\%; 95\% CI 5.3 - 6.6). So it is possible to expect a lengthened recovery of hemoglobin levels after CS. Recent findings [16] show that In contrast with type of breastfeeding, maternal anemia did have an influence on the hemoglobin levels of 6-month-old infants, even when only children on "exclusive + predominant breastfeeding" were analyzed. These findings highlight the need to prevent maternal anemia before conception, during pregnancy and most importantly throughout lactation. Even though our study lacks data of babies hemoglobin levels, their general health and weight gain may indicate better ferrous metabolism of mothers on LNG-IUS group.

\section{CONCLUSION}

In the present study, the contraceptive efficacy of both IUDs was high, with an acceptable safety profile. Our data seem to confirm previous observation with the insertion of LNG-IUS during CS mainly prolonging amenorrhea resulting in faster recovery of ferrous homeostasis. Due to the increased blood loss after cesarean section we believe this is a clear advantage of LNGIUS $^{\circledR}$ over Copper T 380 A use.

\section{REFERENCES}

[1] Kapp, N. and Curtis, K. (2009) Intrauterine device insertion during the postpartum period: A systematic review. Contraception, 80, 327-336. doi:10.1016/j.contraception.2009.03.024

[2] LNG-IUS $^{\circledR}$ (Levonorgestrel-Releasing Intrauterine System) prescribing information. Berlex Laboratories, Inc., Montville. http://www.LNG-IUS-us.com/pdf/LNG-IUS-pi.pdf

[3] Shaamash, A.H., Sayed, G.H., Hussein, M.M. and Shaaban, M.M. (2005) A comparative study of the levonorgestrelreleasing intrauterine system LNG-IUS versus the Copper T 380 A intrauterine device during lactation: Breastfeeding performance, infant growth and infant development. Contraception, 72, 346-351. doi:10.1016/j.contraception.2005.04.004

[4] Puzey, M. (2005) LNG-IUS at caesarean section. European Journal of Contraception Reproductive Health Care, 10, 165-167.

[5] Family Health International (1985) Postpartum IUDs in Mexico. Network, 6, 8.

[6] Chi, I.-C., Ji, G., Siemens, A.J. and Waszak, C.S. (1986) IUD insertion at cesarean section-The Chinese experience. Advances in Contraception, 2, 145-153. doi:10.1007/BF01849224

[7] Chi, I.-C., Zhou, S.-W., Balogh, S. and Ng, K. (1984) 
Post-cesarean section insertion of intrauterine devices. American Journal of Public Health, 74, 1281-1282. doi:10.2105/AJPH.74.11.1281

[8] Van Kets, H., Thiery, M. and Van der Pas, H. (1985) IUD insertion during cesarean section. Advances in Contraception, 1, 337-340. doi:10.1007/BF01849309

[9] Lara, R., Sanchez, R.A. and Aznar, R. (1989) IUD insertion during cesarean section (Aplicacion del dispositivo intrauterino a traves de la incision de la cesarea). Ginecologia y Obstetricia de Mexico, 57, 23-27.

[10] Xu, J.-X., Connell, C. and Chi, I.-C. (1992) Immediate postpartum intrauterine device insertion. A report on the Chinese experience. Advances in Contraception, 8, 281290. doi:10.1007/BF02042586

[11] Chi, I.-C. (1985) IUD use in diabetic or lactating women or women after cesarean delivery-An epidemiologic perspective. Advances in Contraceptive Delivery Systems (Monograph), 2, 2287-2297.

[12] Cole, L.P, Edelman, D.A., Potts, D.M., Wheeler, R.G. and Laufe, L.E. (1984) Postpartum insertion of modified intrauterine devices. Journal of Reproductive Medicine, 29,
677-682.

[13] Heikkilä, M., Haukkamaa, M. and Luukkainen, T. (1984) Contraception with a levonorgestrel-releasing IUD: Effect on lactation and child development. In: Zatuchni, G.I., Goldsmith, A., Shelton, J.D., Sciarra, J.J. and Osborn, C.K., Eds., Long-Acting Contraceptive Delivery Systems, Harper \& Row, Philadelphia, 368-377.

[14] Luukkainen, T., Lahteenmaki, P. and Toivonen, J. (1990) Levonorgestrel-releasing intrauterine device. Annals of Medicine, 22, 85-90. doi:10.3109/07853899009147248

[15] Rossen, J., Økland, I., Nilsen, O.B. and Eggebø, T.M. (2010) Is there an increase of postpartum hemorrhage, and is severe hemorrhage associated with more frequent use of obstetric interventions? Acta Obstetricia et Gynecologica, 89, 1248-1255. doi:10.3109/00016349.2010.514324

[16] Teixeira, P.D, Liran, P.I., Coutinho, S.B., Eickmann, S.H. and Lima, M.C. (2010) Influence of breastfeeding type and maternal anemia on hemoglobin concentration in 6month-old infants. Jornal de Pediatria, 86, 65-72. doi:10.2223/JPED.1959 